Published in final edited form as:

J Phys Chem Lett. 2016 September 1; 7(17): 3365-3370. doi:10.1021/acs.jpclett.6b01512.

\title{
Submillisecond Dynamics of Mastoparan X Insertion into Lipid Membranes
}

\author{
Erin E. Schuler, Sureshbabu Nagarajan, and R. Brian Dyer \\ Department of Chemistry, Emory University, Atlanta, Georgia 30322-1005, United States
}

\begin{abstract}
The mechanism of protein insertion into a lipid bilayer is poorly understood because the kinetics of this process is difficult to measure. We developed a new approach to study insertion of the antimicrobial peptide Mastoparan X into zwitterionic lipid vesicles, using a laser-induced temperature-jump to initiate insertion on the microsecond time scale and infrared and fluorescence spectroscopies to follow the kinetics. Infrared probes the desolvation of the peptide backbone and yields biphasic kinetics with relaxation lifetimes of 12 and $117 \mu \mathrm{s}$, whereas fluorescence probes the intrinsic tryptophan residue located near the $\mathrm{N}$-terminus and yields a single exponential phase with a lifetime of $440 \mu \mathrm{s}$. Arrhenius analysis of the temperature-dependent rates yields an activation energy for insertion of $96 \mathrm{~kJ} / \mathrm{mol}$. These results demonstrate the complexity of the insertion process and provide mechanistic insight into the interplay between peptides and the lipid bilayer required for peptide transport across cellular membranes.
\end{abstract}

\section{Graphical abstract}

*Corresponding Author: Address: Emory University, Department of Chemistry, 1515 Dickey Drive, Atlanta, GA 30322. Phone: 404-727-6637; briandyer@emory.edu.

Notes

The authors declare no competing financial interest.

Supporting Information

The Supporting Information is available free of charge on the ACS Publications website at DOI: 10.1021/acs.jpclett.6b01512.

Detailed experimental materials and methods and supporting data including MPX circular dichroism and FTIR spectra and melts, vesicle leakage assay, van't Hoff analysis of temperature dependent equilibrium data, T-jump kinetics of gel to fluid phase transition, and determination of bimolecular association rates (PDF) 


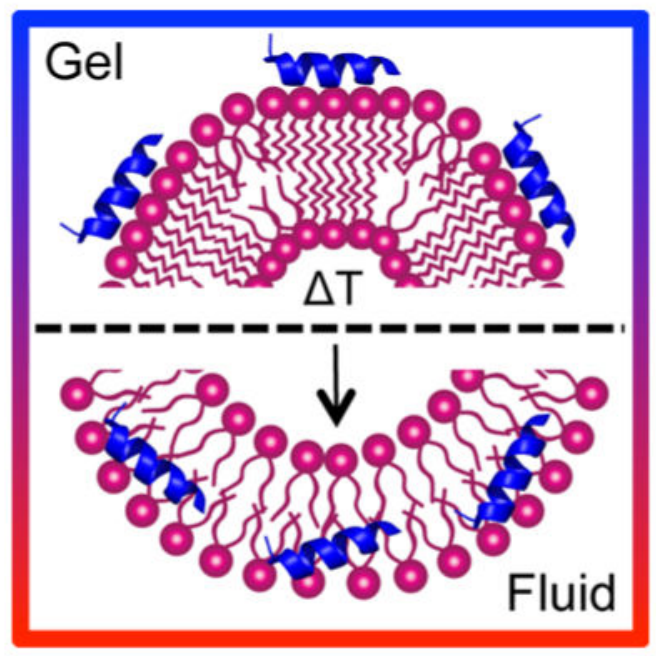

The mechanism of protein insertion into membranes is poorly understood despite being critical to a wide range of cellular functions, including cellular signaling and communication, membrane protein folding, membrane fusion, viral infectivity, and a host of others. ${ }^{1-4}$ Protein insertion into a membrane is usually coupled to folding, since nascent membrane proteins are typically transported unstructured and only develop their functional structures by interacting with the target membrane. Past studies of peptide insertion and coupled folding reactions have relied on stopped flow mixing experiments $;{ }^{5}$ however, this methodology is limited by a classic kinetic quandary. Since the slowest step is usually the diffusion and association of the peptide to the membrane surface, the kinetics of faster subsequent steps such as insertion cannot be observed. Many dynamic processes of both the membrane and the peptide occur in the submillisecond time regime and therefore cannot be accessed in stopped flow mixing experiments. Therefore, a new approach is required to resolve the dynamics of peptide insertion, the coupled dynamics of the membrane, and any subsequent folding or oligomerization steps. ${ }^{6}$

Some small linear peptides such as antimicrobial peptides (AMPs) are capable of spontaneous insertion into membranes. AMPs are typically short sequences of amino acids that yield amphipathic secondary structures with a net cationic charge. ${ }^{7-9}$ Many AMPs require direct interaction with target membranes to fold to their native structures in both naturally occurring and synthetic membrane systems. ${ }^{10}$ These properties of AMPs make them ideal model systems to study the dynamics of peptide membrane interactions. We investigated the mechanism of peptide insertion of the AMP Mastoparan X (MPX), into model deuterated 1,2-dipalmitoyl-sn-glycero-3-phospho-choline (d62-DPPC) lipid vesicles. MPX is a 14-residue peptide (INWKGIAAMAKKLL-NH ${ }_{2}$ ) isolated from wasp venom that carries a $4^{+}$charge at neutral $\mathrm{pH} \cdot{ }^{11,12}$ Although unstructured in an aqueous environment, MPX folds to adopt an $a$-helical conformation in the presence of both negatively charged and zwitterionic membranes. ${ }^{11,13}$ The orientation of MPX in the membrane has been shown to depend on the surface charge of the membrane. ${ }^{14,15}$ Previous studies that examined the folding and insertion of MPX and similar peptides found a tightly coupled relationship between the peptide folding and the ability to traverse membranes. ${ }^{16-19}$ 
MPX spontaneously inserts into a fluid membrane. In the present work we explore whether it is possible to control this process by manipulating membrane order to stabilize the surface associated state of the peptide. We find that in the rigid gel phase of the saturated DPPC membrane, MPX binds to the surface of the membrane with minimal insertion, apparently because the compact membrane structure presents a substantial barrier to insertion. Subsequent to equilibration of MPX with the surface, insertion into the membrane is triggered by rapid thermal initiation of the gel to fluid phase transition using a laser-induced T-jump. We previously characterized the gel to fluid phase transition of model DPPC membranes through T-jump experiments. ${ }^{20} \mathrm{We}$ found that the bulk of the phase transition is accomplished in less than $1 \mu \mathrm{s}$, followed by a slower equilibration of water through the membrane. Therefore, initiating insertion by control of the membrane fluidity provides sufficient time resolution to capture the dynamics of interest by avoiding mixing and diffusion processes. The results indicate that MPX insertion into the lipid bilayer is a complex process that is significantly faster than previously reported.

CD spectroscopy was used to confirm proper folding of the purified MPX and thermal stability in the presence of d62-DPPC SUVs (Supporting Information (SI) Figure S1). In the presence of d62-DPPC membrane vesicles at $20{ }^{\circ} \mathrm{C}$, the $\mathrm{CD}$ spectrum exhibits dual minima at 208 and $222 \mathrm{~nm}$ characteristic of an $\boldsymbol{a}$-helix. This temperature is well below the gel-tofluid phase transition $\left(T_{\mathrm{m}}=37^{\circ} \mathrm{C}\right)$ of the membrane. The fluorescence behavior of MPX above and below the membrane fluid phase transition was also characterized. MPX contains a tryptophan residue that acts as an intrinsic fluorescent probe, sensitive to changes in structure and solvation. Addition of d62-DPPC SUVs to a solution of MPX causes a dramatic blue shift of the fluorescence maximum, from 346 to $327 \mathrm{~nm}$, as shown in Figure 1A. This blue shift is characteristic of sequestration of Trp from solvent water, for example as observed in protein folding. ${ }^{21}$ The inset of Figure 1A shows the temperature dependence of the integrated Trp fluorescence band. The fluorescence exhibits a sharp increase near $T_{\mathrm{m}}$ of the gel to fluid phase transition of the membrane, consistent with insertion of the peptide triggered by increasing fluidity of the membrane. Temperature-dependent FTIR absorbance spectra of MPX and d62-DPPC LUVs (SI Figure S2) provide additional insight into the response of MPX and the membrane to a temperature change. The induced changes of the amide I mode of the peptide backbone and both the ester $\mathrm{C}=\mathrm{O}$ stretch of the lipid headgroup and the $\mathrm{CD}_{2}$ stretch of the lipid tails are highlighted in the temperature-dependent FTIR difference spectra (Figure 1B). The peptide amide I region of these difference spectra reveals a new absorbance that grows in at $1655 \mathrm{~cm}^{-1}$ and a concomitant bleach at $1634 \mathrm{~cm}^{-1}$. Previous studies have assigned the $1634 \mathrm{~cm}^{-1}$ peak to solvated helix and the higher frequency $1655 \mathrm{~cm}^{-1}$ peak to solvent protected or "buried" helix. ${ }^{22,23}$ Together the CD, fluorescence, and IR data support the model shown in Scheme 1, in which MPX is folded and bound to the membrane surface as a solvated helix at lower temperatures, then inserted into the membrane core and protected from solvent at higher temperatures. These results are also consistent with previous NMR and IR studies of the structure of MPX inserted in a fluid membrane bilayer, which found that it lies at a $14^{\circ}$ angle to the membrane surface with the $\mathrm{N}$-terminus closer to the interfacial region of the membrane and one face more fully buried in the hydrophobic core. ${ }^{14,15}$ 
Since the equilibrium studies demonstrate that the peptide associates to the membrane surface in the gel phase with minimal insertion, it should be possible to rapidly trigger insertion using a laser-induced T-jump and thus observe the insertion kinetics faster than the diffusion limit. We demonstrated previously that the membrane phase transition occurs within a few hundred nanoseconds, thus the T-jump produces conditions that favor insertion on this time scale. T-jump IR data show little perturbation of lipid dynamics in the presence of MPX (SI Figure S4, Table S2). The infrared and fluorescence spectra of the peptide provide clear signatures for insertion that can be used to probe the time dependence of this process. T-jump Trp fluorescence transients for T-jumps from different starting temperatures are shown in Figure 2A.

The relaxation lifetime of insertion was determined by fitting the kinetic traces to a single exponential, revealing burial of Trp into the membrane within hundreds of microseconds (Figure 2A, Table 1). Insertion of MPX results in protection of the Trp residue from solvent quenching interactions, ${ }^{21}$ thus generating the observed positive transient emission signal. Arrhenius analysis of the resulting rates yielded an activation energy $\left(E_{\mathrm{a}}\right)$ of $96 \mathrm{~kJ} / \mathrm{mol}$ (Figure 2A inset). No transient emission is observed for the first kinetic trace, corresponding to a jump from 15 to $22.9^{\circ} \mathrm{C}$. This observation is consistent with a model where MPX acquires entry into the membrane by insertion into sites having more fluid-like lipid properties. For the jump to $23{ }^{\circ} \mathrm{C}$, the d62-DPPC membrane is still in the gel-phase and the MPX is unable to insert. Table 1 summarizes the amplitude of transient emission, which increases as the temperature-jump approaches the main transition of the membrane, indicating an increase in peptide insertion correlated with the increase in membrane fluidity.

To complement the T-jump fluorescence data, insertion of the amide $\mathrm{I}^{\prime}$ backbone into the membrane was also probed by T-jump infrared. The buried helix band at $1650 \mathrm{~cm}^{-1}$ was used as the probe frequency to capture the dynamics of dehydration of the amide $\mathrm{I}^{\prime}$ backbone as MPX inserts into the lipid bilayer. A frequency of $1600 \mathrm{~cm}^{-1}$ was selected as the reference because it is associated with minimal signal from the amide backbone in the equilibrium FTIR measurements. Monitoring the $1650 \mathrm{~cm}^{-1}$ band for a T-jump from 28 to $36{ }^{\circ} \mathrm{C}$ produces transient absorbance data that can be modeled to a biexponential fit, corresponding to lifetimes of 12 and $117 \mu$ s (Figure 2B). Around $1 \mathrm{~ms}$, the sample begins to cool, and the transient absorbance recovers back to the baseline within $10 \mathrm{~ms}$. The faster phase is clearly observed in the T-Jump IR experiments, but not observed in the transient fluorescence data, indicating that the IR probe is uniquely sensitive to this initial process. This fast phase may be attributed to one of several possible events related to the insertion of MPX. One possibility is that subsequent to membrane association and folding, insertion requires rearrangement of the amphipathic helix on the surface of the membrane. For example, insertion may require rotation of the hydrophobic face of the peptide to facilitate burial into the hydrophobic membrane core. This phase could also be attributed to an intermediate structure, e.g., MPX that is partially dehydrated and inserted. Previous studies of the interaction of the pHLIP peptide with membranes support the possibility of heterogeneous conformations of the peptide on the membrane surface. ${ }^{25}$ If insertion of MPX were directional, this intermediate would not necessarily be detected by fluorescence because the Trp probe is locally reporting near one end of the peptide. 
The second, slower phase of the transient IR data harbors the majority of the amplitude and thus is the major reporter on insertion. This phase corresponds to the single kinetics phase observed in the T-jump fluorescence experiment. Interestingly, the observed rate is almost 4 times faster than the rate observed in the transient fluorescence experiments. If the insertion were simply two-state state (in or out), with a single barrier between them, the observed relaxation lifetime would be the same regardless of the probe. Therefore, these results indicate that there is either an intermediate representing a partially inserted peptide or a distribution of barriers that may be the result of an inhomogeneously solvated backbone. Further insight can be gained by considering the nature of each spectroscopic probe. Tryptophan is a local probe and senses insertion near the N-terminus. By contrast, the IR probe is the helix component of the amide I' band, which reports on the global peptide backbone. Therefore, the IR transient gives an average rate of burial of the entire helix. This average lifetime is faster than the burial of the Trp located near the N-terminus, an observation that suggests that the insertion of MPX is vectorial, where the C-terminus is inserted first. Since the equilibrium structure of inserted MPX is nearly parallel to the membrane surface, with the C-terminus more deeply buried, this suggests that the peptide penetrates the membrane interface at a small angle and submarines into the core. Directional insertion controlled by the $\mathrm{C}$-terminus of the peptide is also supported by the structure of MPX since the $\mathrm{N}$-terminus is positively charged, whereas the $\mathrm{C}$-terminus is amidated and uncharged. Vectorial C-terminal insertion has been observed for related peptide systems such as the pHLIP family of flanking and TM sequences. ${ }^{26}$

The T-jump results indicate that the MPX insertion lifetime is an order of magnitude shorter than the millisecond lifetime previously reported for this process. We attribute this discrepancy to experimental limitations of stopped flow methods previously used to measure insertion kinetics. Mixing methods are intrinsically limited by the diffusion rate of the peptide to the membrane surface. In addition, the dead time of the stopped flow apparatus represents a technical limit of this approach. In contrast, transient measurements acquired by T-jump provide a significant enhancement in time resolution (20 ns), while simultaneously providing a trigger to thermally induce the gel to fluid phase transformation of the membrane, thereby promoting MPX insertion. Previous studies investigated insertion with various starting conditions, including an unfolded, preassociated state, ${ }^{24}$ a prefolded solvated state, ${ }^{25}$ or an unfolded unassociated state in which folding occurs concomitant with membrane binding. ${ }^{27}$ In these instances, insertion is initiated by stopped-flow mixing to either promote the association of the peptide to the membrane, or using a $\mathrm{pH}$ jump, to trigger peptide folding and subsequent insertion. In the latter example, initiating folding from an unfolded preadsorbed state slowed the apparent insertion rate due to burial of titratable residues in the interfacial region of the bilayer. ${ }^{25}$ In comparison, our studies offer an alternative scenario where we investigate insertion starting from a state where the peptide is (1) associated with the membrane and (2) already folded at the point of triggering the insertion. The ability to thermally control membrane rigidity and therefore permeability allows for equilibration of the folded peptide with the membrane surface prior to insertion, avoiding the experimental limitations that arise from mixing and diffusion. The observed rate of MPX insertion is also about an order of magnitude faster than the rate predicted by MD simulations for GL8, a helical peptide of similar size to MPX. ${ }^{28}$ The MD simulations 
predict an insertion time of about $9 \mathrm{~ms}$ at $30{ }^{\circ} \mathrm{C}$ with a calculated activation energy of about $84 \mathrm{~kJ} \mathrm{~mol}^{-1}$. It is important to note, however, that the simulations were conducted at high temperature to speed up the kinetics and thus reduce the computational time to a tractable level. The predicted kinetics near room temperature represent an extrapolation of over 5 orders of magnitude in rate, which may introduce significant error.

The T-jump experiments also define the energetic barrier governing MPX insertion. Arrhenius analysis of the temperature dependent rates of insertion yields an activation energy of $96 \mathrm{~kJ} / \mathrm{mol}$. There are two likely contributions to this activation energy, based on the established unfavorable energetics of partitioning a peptide from solvent into the membrane: dehydration of the peptide backbone and partitioning non-hydrogen bonded peptide bonds into the membrane. ${ }^{1}$ Although the partitioning energetics are derived from equilibrium studies, they provide a basis for estimating the maximum possible contribution of specific molecular processes to the kinetic barrier. The best estimate of the energetic cost of dehydration of the helix backbone upon insertion into the hydrophobic core of the lipid bilayer is $5 \mathrm{~kJ} \mathrm{~mol}^{-1}$ per residue. ${ }^{29}$ Since the MPX is bound to the membrane surface before insertion, it is already partially dehydrated; assuming only the residues that are solvent exposed contribute, the maximum possible contribution to the activation energy from dehydration would be $35 \mathrm{~kJ} \mathrm{~mol}^{-1}$. Computational studies estimate the partitioning free energy for transfer of a non-hydrogen bonded peptide bond from water to alkane ( $\left.\Delta G_{\mathrm{CONH}}\right)$ is $26.8 \mathrm{~kJ} \mathrm{~mol}^{-1}$ and from the membrane interface to alkane about half that amount. ${ }^{30}$ From the NMR structure of MPX, ${ }^{13}$ there are three non-hydrogen bonded amide groups at the Cterminus, two of which are oriented toward the interface for the amphipathic helix bound to the membrane surface (the terminal leucines). Therefore, burial of the non-hydrogen bonded amide groups gives a maximum possible contribution to the activation energy of $54 \mathrm{~kJ}$ $\mathrm{mol}^{-1}$. Taken together, the maximum possible contribution from these two processes does not completely account for the measured activation energy; furthermore, this analysis assumes the transition state is completely desolvated, which is highly unlikely. There must be additional contributions to the observed activation energy, probably involving the rearrangement of the lipid bilayer.

In summary, we have exploited membrane fluidity to control the insertion of a peptide into the lipid bilayer, and thus resolved the rapid dynamics of this process for the first time. The dependence of insertion on membrane fluidity is consistent with a wide range of known cell membrane phenomena, including the modulation of protein dynamics ${ }^{31}$ and thermal sensing and signaling. ${ }^{32,33}$ Using a combination of spectroscopic probes, we have shown that the rigid, close-packed structure of saturated membranes in the gel phase allows for binding of solvated MPX on the membrane surface without significant insertion. MPX insertion was then triggered by a laser-induced T-jump to rapidly initiate the transition to the fluid phase of the d62-DPPC vesicle. The dynamics of the insertion process was determined using multiple spectroscopic probes, which reveal the complexity of the insertion process. This unique approach may be widely applicable to other membrane inserting peptides and even whole proteins. 


\section{MATERIALS AND METHODS}

\section{Sample Preparation}

Mastoparan X was synthesized by standard Fmoc solid phase peptide synthesis on a CEM Liberty 1 peptide synthesizer using a rink-amide resin. Purification of MPX was completed on a reverse phase $\mathrm{C} 18$ column (Phenomenex, Torrance, $\mathrm{CA}$ ) with $\mathrm{HCl}$ as a counterion using a linear gradient of water and acetonitrile. The mass of the purified peptide (1557 amu) was confirmed by MALDI-MS. Deuterated d62-DPPC lipids in chloroform and mini-extruder kit were purchased from Avanti Polar Lipid, Inc. LUVs were prepared by sample hydration followed by the extrusion method. SUVs used in the fluorescence T-jump studies were prepared by the same sample hydration method; however, instead of extrusion, the samples were subjected to $45 \mathrm{~min}$ of sonication in a bath sonicator. The peptide was cold mixed at $4{ }^{\circ} \mathrm{C}$ with the vesicle solution (to prevent peptide insertion) to yield a peptide: lipid ratio of 1:16.5. This ratio was selected based on previous NMR studies probing the structure and orientation of MPX on lipid membranes. ${ }^{15}$

\section{Equilibrium Spectroscopy}

Temperature-dependent CD spectroscopy was performed on a JASCO J-810 spectropolarimeter equipped with a PFD-425S Jasco temperature controller (Jasco, Inc., Easton, MD) in a quartz cuvette with a $1 \mathrm{~mm}$ path length. Temperature-dependent fluorescence measurements were obtained on a Dual-FL spectrophotometer (Horiba Scientific, Edison, New Jersey) with a Peltier thermostat to control the cuvette temperature to within $0.1{ }^{\circ} \mathrm{C}$. The MPX peptide was dissolved in $20 \mathrm{mM}$ sodium phosphate buffer at $\mathrm{pH}$ 7.4 to a concentration of $75 \mu \mathrm{M}$ either in the presence or absence of d62-DPPC lipid vesicles (1.24 mM concentration, yielding a 1:16.5 peptide-to-lipid molar ratio). Temperaturedependent equilibrium FTIR spectra were measured on a Varian 3100 Excalibur FTIR spectrophotometer equipped with a liquid nitrogen cooled mercury cadmium telluride detector. Sample and reference solutions were prepared in $\mathrm{D}_{2} \mathrm{O}$ with matching conditions ( $20 \mathrm{mM}$ sodium phosphate buffer, $100 \mathrm{mM} \mathrm{NaCl}$, uncorrected $\mathrm{pH}$ meter reading of 7.4) but with the sample containing 1.5 mM MPX and $24 \mathrm{mM}$ d62-DPPC, a 16:1 lipid:MPX ratio and loaded into a split IR cell with a $126 \mu \mathrm{m}$ Teflon spacer between two $\mathrm{CaF}_{2}$ windows held in a copper housing.

\section{T-Jump Fluorescence and Infrared}

The T-jump apparatus used to measure peptide insertion and lipid relaxation in this study has been described previously. ${ }^{34,35}$ Briefly, this is a pump probe experiment with a near-IR pump laser (Q-switched Ho:YAG at $1908 \mathrm{~nm}$ ) to produce the T-jump and a UV probe laser (frequency tripled Ti:sapphire at $285 \mathrm{~nm}$ ) to excite the time-dependent fluorescence or a Quantum Cascade Laser to monitor the time-dependent mid-IR absorption. The same $\mathrm{CaF}_{2}$ split cell used in the equilibrium FTIR measurements was used for kinetic measurements of both the sample and reference. For the fluorescence T-jump measurements, deuterium exchanged MPX in $\mathrm{NaHPO}_{4}$ buffer without membrane was used as the reference. The temperature-dependent fluorescence of the reference MPX solution (without the membrane) serves as an internal thermometer to determine the magnitude of the T-jump and hence the final temperature. Fluorescence T-jump transients were generated by subtracting the change 
in emission of MPX without membrane in response to a T-jump, from emission generated from MPX in the presence of d62-DPPC to correct for the loss of fluorescence signal that follows the T-jump (on the $10 \mathrm{~ns}$ time scale), due to the temperature-dependent decrease in intrinsic quantum yield of the Trp emission. T-jump infrared transients were obtained using the d62-DPPC vesicles in $\mathrm{D}_{2} \mathrm{O} \mathrm{NaH} \mathrm{PO}_{4}$ buffer as the reference. The temperaturedependent $\mathrm{D}_{2} \mathrm{O}$ absorbance acts as an internal thermometer to determine the magnitude of the T-jump and hence the final temperature. The kinetic traces were generated by subtracting the change in absorbance of the reference at $1600 \mathrm{~cm}^{-1}$ from the $1650 \mathrm{~cm}^{-1}$ sample frequency of the lipid-peptide mixture in response to the T-jump.

\section{Supplementary Material}

Refer to Web version on PubMed Central for supplementary material.

\section{Acknowledgments}

This work was supported by National Institutes of Health Grant GM053640.

\section{References}

1. Cymer F, von Heijne G, White SH. Mechanisms of Integral Membrane Protein Insertion and Folding. J Mol Biol. 2015; 427:999-1022. [PubMed: 25277655]

2. Martens S, McMahon HT. Mechanisms of Membrane Fusion: Disparate Players and Common Principles. Nat Rev Mol Cell Biol. 2008; 9:543-556. [PubMed: 18496517]

3. Lorenz D, Wiesner B, Zipper J, Winkler A, Krause E, Beyermann M, Lindau M, Bienert M. Mechanism of Peptide-Induced Mast Cell Degranulation: Translocation and Patch-Clamp Studies. J Gen Physiol. 1998; 112:577-591. [PubMed: 9806967]

4. Cho, WH.; Stahelin, RV. Annual Reviews Biophysics and Biomolecular Structure; Annual Reviews. Vol. 34. Palo Alto, CA: 2005. Membrane-Protein Interactions in Cell Signaling and Membrane Trafficking; p. 119-151.

5. Tang J, Signarvic RS, DeGrado WF, Gai F. Role of Helix Nucleation in the Kinetics of Binding of Mastoparan X to Phospholipid Bilayers. Biochemistry. 2007; 46:13856-13863. [PubMed: 17994771]

6. Oh KI, Smith-Dupont KB, Markiewicz BN, Gai F. Kinetics of Peptide Folding in Lipid Membranes. Biopolymers. 2015; 104:281-90. [PubMed: 25808575]

7. Epand RM, Vogel HJ. Diversity of Antimicrobial Peptides and Their Mechanisms of Action. Biochim Biophys Acta, Biomembr. 1999; 1462:11-28.

8. Hancock REW, Sahl HG. Antimicrobial and Host-Defense Peptides as New Anti-Infective Therapeutic Strategies. Nat Biotechnol. 2006; 24:1551-1557. [PubMed: 17160061]

9. Hancock REW, Rozek A. Role of Membranes in the Activities of Antimicrobial Cationic Peptides. FEMS Microbiol Lett. 2002; 206:143-149. [PubMed: 11814654]

10. Sitaram N, Nagaraj R. Interaction of Antimicrobial Peptides with Biological and Model Membranes: Structural and Charge Requirements for Activity. Biochim Biophys Acta, Biomembr. 1999; 1462:29-54.

11. Higashijima T, Wakamatsu K, Takemitsu M, Fujino M, Nakajima T, Miyazawa T. Conformational Change of Mastoparan from Wasp Venom on Binding with Phospholipid Membrane. FEBS Lett. 1983; 152:227-230. [PubMed: 6825849]

12. Okamura K, Inui K, Hirai Y, Nakajima T. The Effect of Mastoparan on Ion Movement in Black Lipid Membrane. Biomed Res. 1981; 2:450-452.

13. Todokoro Y, Yumen I, Fukushima K, Kang SW, Park JS, Kohno T, Wakamatsu K, Akutsu H, Fujiwara T. Structure of Tightly Membrane-Bound Mastoparan-X, a G-Protein-Activating Peptide, Determined by Solid-State NMR. Biophys J. 2006; 91:1368-1379. [PubMed: 16714348] 
14. Tucker MJ, Getahun Z, Nanda V, DeGrado WF, Gai F. A New Method for Determining the Local Environment and Orientation of Individual Side Chains of Membrane-Binding Peptides. J Am Chem Soc. 2004; 126:5078-5079. [PubMed: 15099085]

15. Whiles JA, Brasseur R, Glover KJ, Melacini G, Komives EA, Vold RR. Orientation and Effects of Mastoparan X on Phospholipid Bicelles. Biophys J. 2001; 80:280-293. [PubMed: 11159401]

16. Hellmann N, Schwarz G. Peptide-Liposome Association. A Critical Examination with Mastoparan-X. Biochim Biophys Acta, Biomembr. 1998; 1369:267-277.

17. Henriksen JR, Andresen TL. Thermodynamic Profiling of Peptide Membrane Interactions by Isothermal Titration Calorimetry: A Search for Pores and Micelles. Biophys J. 2011; 101:100-109. [PubMed: 21723819]

18. Schwarz G, Blochmann U. Association of the Wasp Venom Peptide Mastoparan with Electrically Neutral Lipid Vesicles. FEBS Lett. 1993; 318:172-176. [PubMed: 8440373]

19. Etzerodt T, Henriksen JR, Rasmussen P, Clausen MH, Andresen TL. Selective Acylation Enhances Membrane Charge Sensitivity of the Antimicrobial Peptide Mastoparan-X. Biophys J. 2011; 100:399-409. [PubMed: 21244836]

20. Nagarajan S, Schuler EE, Ma K, Kindt JT, Dyer RB. Dynamics of the Gel to Fluid Phase Transformation in Unilamellar DPPC Vesicles. J Phys Chem B. 2012; 116:13749-13756. [PubMed: 23130986]

21. Eftink MR, Ghiron CA. Exposure of Tryptophanyl Residues and Protein Dynamics. Biochemistry. 1977; 16:5546-5551. [PubMed: 921949]

22. Brewer SH, Tang Y, Vu DM, Gnanakaran S, Raleigh DP, Dyer RB. Temperature Dependence of Water Interactions with the Amide Carbonyls of Alpha-Helices. Biochemistry. 2012; 51:5293-9. [PubMed: 22680405]

23. Gilmanshin R, Dyer RB, Callender RH. Structural Heterogeneity of the Various Forms of Apomyoglobin: Implications for Protein Folding. Protein Sci. 1997; 6:2134-2142. [PubMed: 9336836]

24. Tang J, Yin H, Qiu J, Tucker MJ, DeGrado WF, Gai F. Using Two Fluorescent Probes to Dissect the Binding, Insertion, and Dimerization Kinetics of a Model Membrane Peptide. J Am Chem Soc. 2009; 131:3816-3817. [PubMed: 19256494]

25. Tang J, Gai F. Dissecting the Membrane Binding and Insertion Kinetics of a pHLIP Peptide. Biochemistry. 2008; 47:8250-2. [PubMed: 18636715]

26. Weerakkody D, Moshnikova A, Thakur MS, Moshnikova V, Daniels J, Engelman DM, Andreev OA, Reshetnyak YK. Family of pH (Low) Insertion Peptides for Tumor Targeting. Proc Natl Acad Sci U S A. 2013; 110:5834-5839. [PubMed: 23530249]

27. Tucker MJ, Tang J, Gai F. Probing the Kinetics of Membrane-Mediated Helix Folding. J Phys Chem B. 2006; 110:8105-8109. [PubMed: 16610913]

28. Ulmschneider JP, Smith JC, White SH, Ulmschneider MB. In Silico Partitioning and Transmembrane Insertion of Hydrophobic Peptides under Equilibrium Conditions. J Am Chem Soc. 2011; 133:15487-15495. [PubMed: 21861483]

29. Jayasinghe S, Hristova K, White SH. Energetics, Stability, and Prediction of Transmembrane Helices. J Mol Biol. 2001; 312:927-934. [PubMed: 11580239]

30. Ben-Tal N, Sitkoff D, Topol IA, Yang AS, Burt SK, Honig B. Free Energy of Amide Hydrogen Bond Formation in Vacuum, in Water, and in Liquid Alkane Solution. J Phys Chem B. 1997; 101:450-457.

31. Booth PJ, Riley ML, Flitsch SL, Templer RH, Farooq A, Curran AR, Chadborn N, Wright P. Evidence That Bilayer Bending Rigidity Affects Membrane Protein Folding. Biochemistry. 1997; 36:197-203. [PubMed: 8993334]

32. Inda ME, Vandenbranden M, Fernández A, de Mendoza D, Ruysschaert JM, Cybulski LE. A Lipid-Mediated Conformational Switch Modulates the Thermosensing Activity of DesK. Proc Natl Acad Sci U S A. 2014; 111:3579-3584. [PubMed: 24522108]

33. Martín M, de Mendoza D. Regulation of Bacillus subtilis Desk Thermosensor by Lipids. Biochem J. 2013; 451:269-275. [PubMed: 23356219] 
34. Williams S, Causgrove TP, Gilmanshin R, Fang KS, Callender RH, Woodruff WH, Dyer RB. Fast Events in Protein Folding: Helix Melting and Formation in a Small Peptide. Biochemistry. 1996; 35:691-697. [PubMed: 8547249]

35. Davis CM, Dyer RB. WW Domain Folding Complexity Revealed by Infrared Spectroscopy.

Biochemistry. 2014; 53:5476-5484. [PubMed: 25121968] 

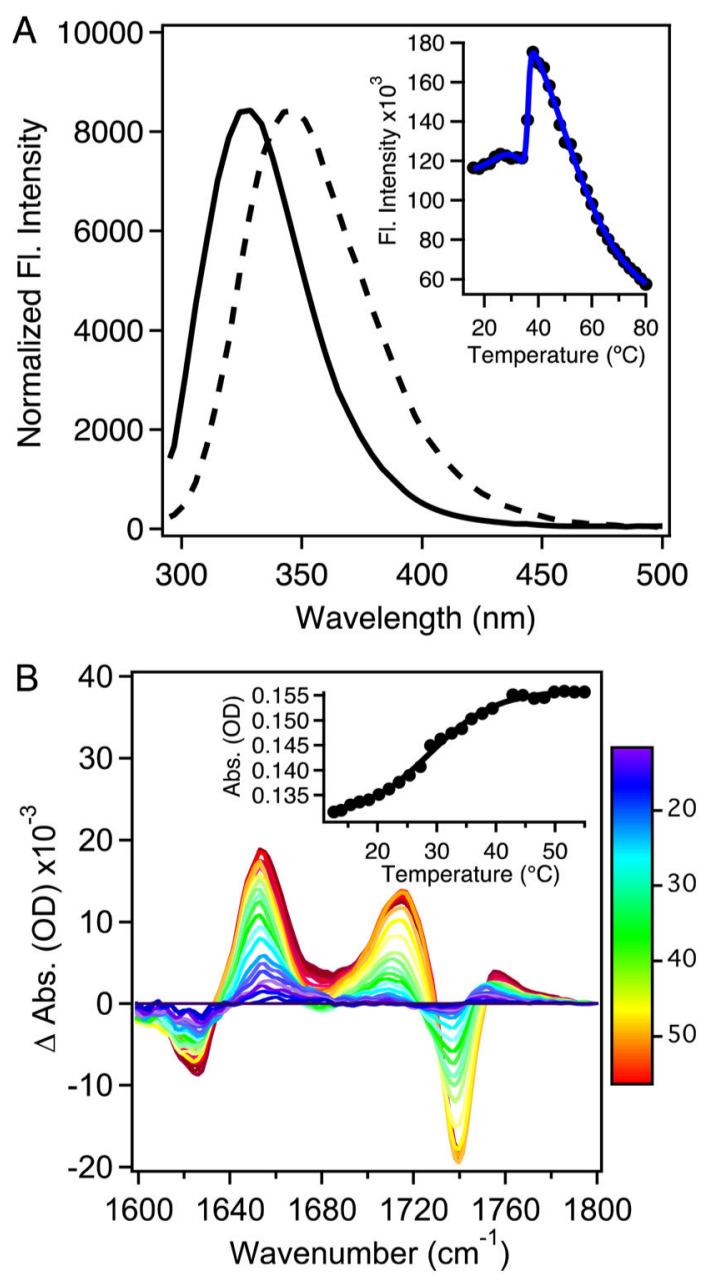

Figure 1.

(A) Normalized tryptophan fluorescence (280 $\mathrm{nm}$ excitation) of $75 \mu \mathrm{M}$ MPX in $20 \mathrm{mM}$ sodium phosphate buffer at $\mathrm{pH} 7.4$, with (solid) and without (dashed) added d62-DPPC SUVs. (Inset) Fluorescence melt obtained by monitoring integrated fluorescence intensity from 327 to $353 \mathrm{~nm}$ as a function of temperature (circles) and fit to 3-state model (line). (B) Temperature-dependent FTIR difference spectra $\left(T-10^{\circ} \mathrm{C}\right)$ in $2{ }^{\circ} \mathrm{C}$ increments of $1.5 \mathrm{mM}$ MPX and d62-DPPC LUVs (16.5:1 lipid:MPX ratio); (inset) IR melt monitored at 1651 $\mathrm{cm}^{-1}$ (amide $\mathrm{I}^{\prime}$ buried helix band). 

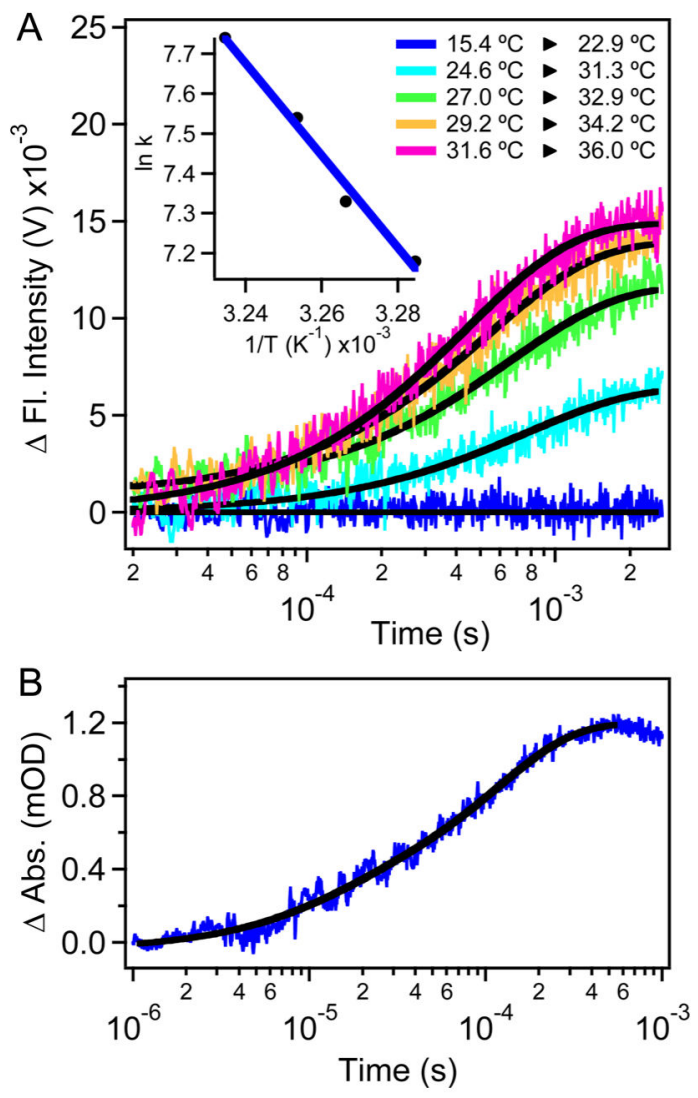

Figure 2.

T-jump transients. (A) T-jump fluorescence transients ( $\lambda_{\mathrm{ex}}=280 \mathrm{~nm}$; emission integrated from 310 to $375 \mathrm{~nm}$ ) and corresponding single exponential fits; (inset) Arrhenius analysis of rates derived from temperature-dependent transient data. (B) T-jump infrared transient for a T-jump from 28 to $36{ }^{\circ} \mathrm{C}$ probed at $1650 \mathrm{~cm}^{-1}$ (buried helix band) and corresponding biexponential fit. 

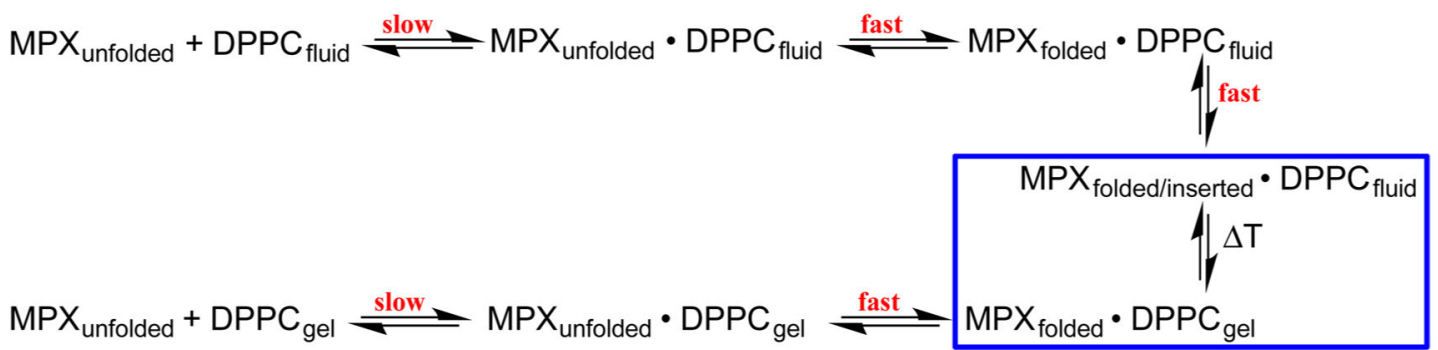

Scheme 1. Proposed MPX Folding/Insertion Pathway ${ }^{a}$

${ }^{a}$ The top and bottom lines represent temperatures above and below the phase transition, respectively, and the blue box represents the insertion process probed in the T-jump measurements. 


\section{Table 1}

\begin{tabular}{|c|c|c|c|}
\hline \multirow{2}{*}{ 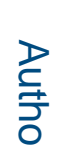 } & \multicolumn{3}{|c|}{ Summary of T-Jump Fluorescence } \\
\hline & T-Jump $\left({ }^{\circ} \mathbf{C}\right)$ & $\tau(\mathbf{s})$ & amplitude $^{a}$ \\
\hline$<$ & $15.4-22.9$ & NA & NA \\
\hline ఏ & $24.6-31.3$ & $7.6 \pm 0.3 \mathrm{e}^{-4}$ & 1.4 \\
\hline $\bar{c}$ & $27.0-32.9$ & $6.5 \pm 0.2 \mathrm{e}^{-4}$ & 2.6 \\
\hline c & $29.2-34.2$ & $5.3 \pm 0.1 \mathrm{e}^{-4}$ & 3.5 \\
\hline & $31.6-36.0$ & $4.4 \pm 0.1 \mathrm{e}^{-4}$ & 3.6 \\
\hline
\end{tabular}

